\title{
Molecular karyotyping in 17 patients and mutation screening in 41 patients with Kabuki syndrome
}

\author{
Hideo Kuniba ${ }^{1,2,14}$, Koh-ichiro Yoshiura ${ }^{1,14}$, Tatsuro Kondoh ${ }^{2}$, Hirofumi Ohashi ${ }^{3,14}$, Kenji Kurosawa ${ }^{4}$, \\ Hidefumi Tonoki ${ }^{5}$, Toshiro Nagai ${ }^{6,14}$, Nobuhiko Okamoto ${ }^{7}$, Mitsuhiro Kato ${ }^{8}$, Yoshimitsu Fukushima ${ }^{9,14}$, \\ Tadashi Kaname ${ }^{10,14}$, Kenji Naritomi ${ }^{10,14}$, Tadashi Matsumoto ${ }^{2}$, Hiroyuki Moriuchi ${ }^{2}$, Tatsuya Kishino ${ }^{11,14}$, \\ Akira Kinoshita $^{1,14}$, Noriko Miyake ${ }^{12,14}$, Naomichi Matsumoto ${ }^{12,14}$ and Norio Niikawa ${ }^{1,13,14}$
}

The Kabuki syndrome (KS, OMIM 147920), also known as the Niikawa-Kuroki syndrome, is a multiple congenital anomaly/ mental retardation syndrome characterized by a distinct facial appearance. The cause of KS has been unidentified, even by whole-genome scan with array comparative genomic hybridization (CGH). In recent years, high-resolution oligonucleotide array technologies have enabled us to detect fine copy number alterations. In 17 patients with KS, molecular karyotyping was carried out with GeneChip 250K Nspl array (Affymetrix) and Copy Number Analyser for GeneChip (CNAG). It showed seven copy number alterations, three deleted regions and four duplicated regions among the patients, with the exception of registered copy number variants (CNVs). Among the seven loci, only the region of 9q21.11-q21.12 ( 1.27 Mb) involved coding genes, namely, transient receptor potential cation channel, subfamily M, member 3 (TRPM3), Kruppel-like factor 9 (KLF9), structural maintenance of chromosomes protein 5 (SMC5) and MAM domain containing 2 (MAMDC2). Mutation screening for the genes detected 10 base substitutions consisting of seven single-nucleotide polymorphisms (SNPs) and three silent mutations in 41 patients with KS. Our study could not show the causative genes for KS, but the locus of 9q21.11-q21.12, in association with a cleft palate, may contribute to the manifestation of KS in the patient. As various platforms on oligonucleotide arrays have been developed, higher resolution platforms will need to be applied to search tiny genomic rearrangements in patients with KS. Journal of Human Genetics (2009) 54, 304-309; doi:10.1038/jhg.2009.30; published online 3 April 2009

Keywords: Kabuki syndrome; microdeletion; molecular karyotyping; mutation screening; Niikawa-Kuroki syndrome

\section{INTRODUCTION}

Kabuki syndrome (KS, OMIM 147920), also known as NiikawaKuroki syndrome, is a multiple congenital anomaly/mental retardation (MCA/MR) syndrome characterized by a distinct facial appearance, skeletal abnormalities, joint hypermobility, dermatoglyphic abnormalities, postnatal growth retardation, recurrent otitis media and occasional visceral anomalies. ${ }^{1,2}$ The prevalence was estimated to be $1 / 32000$ in $\mathrm{Japan}^{3}$ and 1/86000 in Australia and New Zealand. ${ }^{4}$ Although most cases were sporadic, at least 14 familial cases have been reported. It is assumed that KS is an autosomal dominant disorder, considering the equal male-to-female ratio of patients and parent-child transmission pattern in some familial cases. ${ }^{5}$
The cause of KS remains unknown, even though at least 400 patients have been diagnosed in a variety of ethnic groups since $1981 .^{3-7}$ Some works have ruled out several loci; for example, 1q32$\mathrm{q} 41,8 \mathrm{p} 22-\mathrm{p} 23.1$ and $22 \mathrm{q} 11$, as candidates for KS. ${ }^{8-13}$ A study of arraybased comparative genomic hybridization $(\mathrm{CGH})$ showed a disruption of the C20orf133(MACROD2) gene by $\sim 250 \mathrm{~kb}$ deletion in a patient with $\mathrm{KS},{ }^{14}$ but the following mutation screening for the gene failed to find a pathogenic base change within exons in 19 other patients with $\mathrm{KS}^{14}$ and in 43 Japanese patients. ${ }^{15}$ Another study of array CGH with $0.5-1.2 \mathrm{Mb}$ resolution reported that $2 \mathrm{q} 37$ deletions were detected in two patients with Kabuki-like features, but their facial features were not typical for KS. ${ }^{16}$ To date, no concordant specific lesion has been

${ }^{1}$ Department of Human Genetics, Nagasaki University Graduate School of Biomedical Sciences, Nagasaki, Japan; ${ }^{2}$ Department of Pediatrics, Nagasaki University School of Medicine, Nagasaki, Japan; ${ }^{3}$ Division of Medical Genetics, Saitama Children's Medical Center, Iwatsuki, Japan; ${ }^{4}$ Division of Medical Genetics, Kanagawa Children's Medical Center, Yokohama, Japan; ${ }^{5}$ Department of Pediatrics, Tenshi Hospital, Sapporo, Japan; ${ }^{6}$ Department of Pediatrics, Dokkyo University School of Medicine Koshigaya Hospital, Koshigaya, Japan; ${ }^{7}$ Department of Planning and Research, Osaka Medical Center and Research Institute for Maternal and Child health, Osaka, Japan; ${ }^{8}$ Department of Pediatrics, Yamagata University School of Medicine, Yamagata, Japan; ${ }^{9}$ Department of Medical Genetics, Shinshu University School of Medicine, Matsumoto, Japan; ${ }^{10}$ Department of Medical Genetics, University of the Ryukyus, Nishihara, Japan; ${ }^{11}$ Division of Functional Genomics, Center for Frontier Life Sciences, Nagasaki University, Nagasaki, Japan; ${ }^{12}$ Department of Human Genetics, Yokohama City University Graduate School of Medicine, Yokohama, Japan; ${ }^{13}$ Research Institute of Personalized Health Sciences, Health Sciences University of Hokkaido, Tobetsu, Japan and ${ }^{14}$ Solution Oriented Research for Science and Technology (SORST), Japan Science and Technology Agency (JST), Tokyo, Japan

Correspondence: Dr K-i Yoshiura, Department of Human Genetics, Nagasaki University Graduate School of Biomedical Sciences, Sakamoto 1-12-4, Nagasaki 852-8523, Japan. E-mail: kyoshi@nagasaki-u.ac.jp

Received 15 January 2009; revised 3 March 2009; accepted 11 March 2009; published online 3 April 2009 
found by whole-genome scan with array $\mathrm{CGH}$ in a bacterial artificial chromosome (BAC) clone with $0.5-1.5 \mathrm{Mb}$ resolution. ${ }^{16-18}$

Chromosomal aberration analysis by high-resolution oligonucleotide array technologies in recent years, called molecular karyotyping, enables us to detect submicroscopic pathogenic copy number alterations, which were undetectable even by BAC array CGH. ${ }^{19,20}$ As not a few MCA/MR syndromes are because of chromosomal copy number aberration, we hypothesize that some sort of microdeletion/ microduplication causes KS. Herein, we report the results of molecular karyotyping in 17 patients using GeneChip 250K array and those of mutation screening of candidate genes in 41 patients with KS in Japan.

\section{MATERIALS AND METHODS}

\section{Subjects}

The subjects for molecular karyotyping consisted of 18 patients (nine girls and nine boys) at entry. The subjects for mutation screening consisted of 41 patients ( 20 girls and 21 boys), including the aforementioned 18 patients. The diagnoses of KS were confirmed by experts of clinical genetics, although written permission for the use of facial photographs in publications was not obtained. These Japanese patients showed a normal karyotype at a 400-band level, and were earlier reported with no pathogenic genome copy number change by $1.5 \mathrm{Mb}$ resolution BAC array CGH. ${ }^{18}$ Genomic DNA was isolated by the standard method from their peripheral blood leukocytes or in part from their lymphoblastoid cell lines. Experimental procedures were approved by the Committee for the Ethical Issues on Human Genome and Gene Analysis at Nagasaki University.

\section{Molecular karyotyping}

DNA oligomicroarray hybridization, using the GeneChip Human Mapping 250K Nsp Array (Affymetrix, Santa Clara, CA, USA), was carried out for 18 patients with KS, following the provided protocol (Affymetrix). Data were analyzed using GTYPE (GeneChip Genotyping Analysis Software) to detect copy number aberration and visualized using CNAG (Copy Number Analyser for GeneChip) version 3. ${ }^{21}$ References for non-paired analysis of CNAG were chosen from eight unrelated individuals of HapMap samples from the Affymetrix website (http://www.affymetrix.com/support/). The resolution of this procedure was estimated as $\sim 30-100 \mathrm{~kb}$. CNAG version 3 was linked with the University of California Santa Cruz (UCSC) genome browser (http:// genome.ucsc.edu/) assembly May 2004, and then its physical position was referred to the data assembly on March 2006 in the UCSC genome browser after adjustment.

\section{Validation of deletion}

Quantitative PCR (qPCR) analysis to validate deletions was run on a LightCycler 480 Real-Time PCR System (Roche Diagnostics, Mannheim, Germany) using an intercalating dye, SYTO9 (Molecular probes, OR, USA), which is an alternative to SYBR green I. ${ }^{22}$ Absolute quantification was carried out using a second derivative max method. A standard curve of amplification efficiency for each set of primers was generated with a serial dilution of genomic DNA. A corrected gene dosage was given as the ratio of a target gene divided by an internal control gene. The copy number was obtained from a calibration under the assumption that the control genome was diploid.

Target genes of copy number aberration were as follows: SUMF1 (for patient K9); MAMDC2 (for patient K16); and CETN1 (for patient K34). The primer sequences of these genes are available in the online supplementary file. Internal control diploid genes were OAZ2 and USP21. Primer sets of the control genes for genomic DNA were selected from the Real Time PCR Primer Sets website (http://www.realtimeprimers.org/). The control genes were confirmed to have no copy number variants on the Database of Genomic Variants (DGV) updated on 26 June 2008 (http://projects.tcag.ca/variation/). BLAST searches confirmed all primer sequences specific for the gene.

Samples were analyzed in triplicate in a 384-well format in a $10 \mu \mathrm{l} \mathrm{final}$ volume containing about $2 \mathrm{ng}$ genomic DNA, $0.5 \mu \mathrm{M}$ forward primer, $0.5 \mu \mathrm{M}$ reverse primer, 0.1 Units TaKaRa ExTaq HS version (TaKaRa, Kyoto, Japan), $1 \times$ PCR buffer, $200 \mu \mathrm{M}$ dNTP and $0.5 \mu \mathrm{M}$ SYTO9. The amplification conditions consisted of an initial denaturation at $95^{\circ} \mathrm{C}$ for $5 \mathrm{~min}$, followed by 45 cycles of

Table 1 Detected genomic copy number aberrations in 17 patients with Kabuki syndrome

\begin{tabular}{|c|c|c|c|c|c|c|c|}
\hline \multirow{2}{*}{ Cytoband } & \multirow{2}{*}{$\begin{array}{l}\text { Patient(s) } \\
\text { ID }\end{array}$} & \multirow{2}{*}{$\begin{array}{l}C N \\
\text { State }\end{array}$} & \multirow{2}{*}{ Length } & \multicolumn{2}{|c|}{ Physical position } & \multirow{2}{*}{ Involving gene(s) } & \multirow{2}{*}{ Concordant loss/gain on DGV } \\
\hline & & & & Start & End & & \\
\hline 3p26.3 & K7 & 1 & $460 \mathrm{~kb}$ & 1435279 & 1895554 & NR & Variation_8235 \\
\hline $3 p 26.2$ & K9 & $1^{a}$ & $205 \mathrm{~kb}$ & 4009368 & 4214847 & SUMF1 & Variation_8973, 8975, 30169 \\
\hline $4 q 13.2$ & K23 & $1^{a}$ & $1.26 \mathrm{Mb}$ & 66329014 & 67591611 & NR & NR \\
\hline $5 q 21.2-q 21.3$ & K22 & 1 & $281 \mathrm{~kb}$ & 104301325 & 104581898 & NR & Variation_3568 \\
\hline $9 q 21.11-q 21.12$ & K16 & $1^{a}$ & $1.27 \mathrm{Mb}$ & 71760296 & 73031176 & TRPM3, KLF9, SMC5, MAMDC2 & NR \\
\hline $14 q 11.2$ & K5 & 1 & $166 \mathrm{~kb}$ & 19336854 & 19502641 & OR4N2, OR4K2, OR4K5, OR4K1 & Variation_0376, 7028, 8094, 9234, 9235 \\
\hline $15 q 11.2$ & $\mathrm{~K} 1, \mathrm{~K} 23$ & 1 & $972 \mathrm{~kb}$ & 19356830 & 20329239 & OR4M2, OR4N4, LOC65D137 & $\begin{array}{l}\text { Variation_0318, 3070, 8265, 9251, } \\
\text { 9254, } 9256\end{array}$ \\
\hline $18 p 11.32$ & K34 & $1^{\mathrm{a}}$ & $35 \mathrm{~kb}$ & 545074 & 580003 & CETN1 & Variation_5044 \\
\hline 20p12.1 & K6 & $1^{\mathrm{a}}$ & $152 \mathrm{~kb}$ & 14993412 & 15145890 & C20orf133 (MACROD2) $)^{\mathrm{b}}$ & NR \\
\hline $4 q 12$ & K5 & 3 & $104 \mathrm{~kb}$ & 54251599 & 54355281 & NR & NR \\
\hline $8 q 11.21$ & K7 & 3 & $171 \mathrm{~kb}$ & 50641101 & 50812548 & NR & Variation_2751, 3731, 8601, 37765 \\
\hline 10p15.2-p15.1 & $\mathrm{K} 5$ & 3 & $142 \mathrm{~kb}$ & 3663600 & 3805292 & NR & NR \\
\hline $13 q 31.1$ & K6 & 3 & $72 \mathrm{~kb}$ & 82451568 & 82523728 & NR & NR \\
\hline $15 q 11.2$ & K7, K9, K12 & 3 & $877 \mathrm{~kb}$ & 19112164 & 19989036 & CXADRP2, POTEB & Variation_3070, 3951, 8784, 30670, etc. \\
\hline $15 q 25.1$ & K9 & 3 & $165 \mathrm{~kb}$ & 76992181 & 77156751 & CTSH, RASGRF1 & Variation_3970, 7073 \\
\hline $16 q 21$ & K13 & 3 & $283 \mathrm{~kb}$ & 58508008 & 58791285 & NR & NR \\
\hline $17 q 12$ & K7 & 3 & $495 \mathrm{~kb}$ & 31428390 & 31923810 & $\begin{array}{l}C C L 3, C C L 4, C C L 3 L 1, C C L 3 L 3, C C L 4 L 1, \\
C C L 4 L 2, T B C 1 D 3 B, T B C 1 D 3 C, T B C 1 D 3 G\end{array}$ & Variation_3142, 4031, 8841, 30824, etc. \\
\hline $22 q 11.22$ & K5, K12 & 3 & $278 \mathrm{~kb}$ & 20907806 & 21186081 & VPREB1, ZNF280B & Variation_5356, 34540 \\
\hline
\end{tabular}

Abbreviations: CN, copy number; DGV, Database of Genomic Variants; NR, no registration in UCSC genes or DGV.

alidated by quantitative PCR.

beleted region was within intron 5 of the C20orf133 (MACROD2) and did not involve any coding exon. ${ }^{15}$ 
denaturation at $95^{\circ} \mathrm{C}$ for $10 \mathrm{~s}$, annealing at $55^{\circ} \mathrm{C}$ for $10 \mathrm{~s}$ and extension at $72{ }^{\circ} \mathrm{C}$ for $15 \mathrm{~s}$ The data were analyzed using LightCycler 480 Basic Software (Roche Diagnostics) and the melting curve was checked to eliminate non-specific products from the reaction.

\section{Mutation screening of candidate genes}

Candidate genes, identified within a detected deletion, consisted of four genes: TRPM3 (NM_001007471 and NM_206946), KLF9 (NM_001206), SMC5 (NM_015110) and MAMDC2 (NM_153267) located at 9q21.12-q21. 11. The entire coding region and splice junctions of the genes were sequenced on an automated sequencer 3130xl (Applied Biosystems, Foster City, CA, USA) using BigDye version 3.1 (Applied Biosystems). Genomic sequences were retrieved from the UCSC genome browser (assembly: March 2006). PCR primers were designed with the assistance of Primer3 (http://frodo.wi.mit.edu/cgi-bin/primer3/ primer3.cgi). The primer sequences are available in the online supplementary file. Resultant electropherograms were aligned using ATGC version 3.0 (Software Development, Tokyo, Japan) and inspected visually to find DNA alterations.

\section{In silico analysis}

Relations among deleted genes were assessed using online software, PANTHER (Protein Analysis Through Evolutionary Relationships, http://www.pantherdb. org), to determine whether the genes involve some developmental pathway or biological process. ${ }^{23}$ The novel synonymous base substitutions found in the mutation screening were examined for their potential activation of the cryptic splice site by comparison between wild-type allele and mutated allele using the GeneSplicer program (http://www.cbcb.umd.edu/software/GeneSplicer/gene_ spl.shtml).
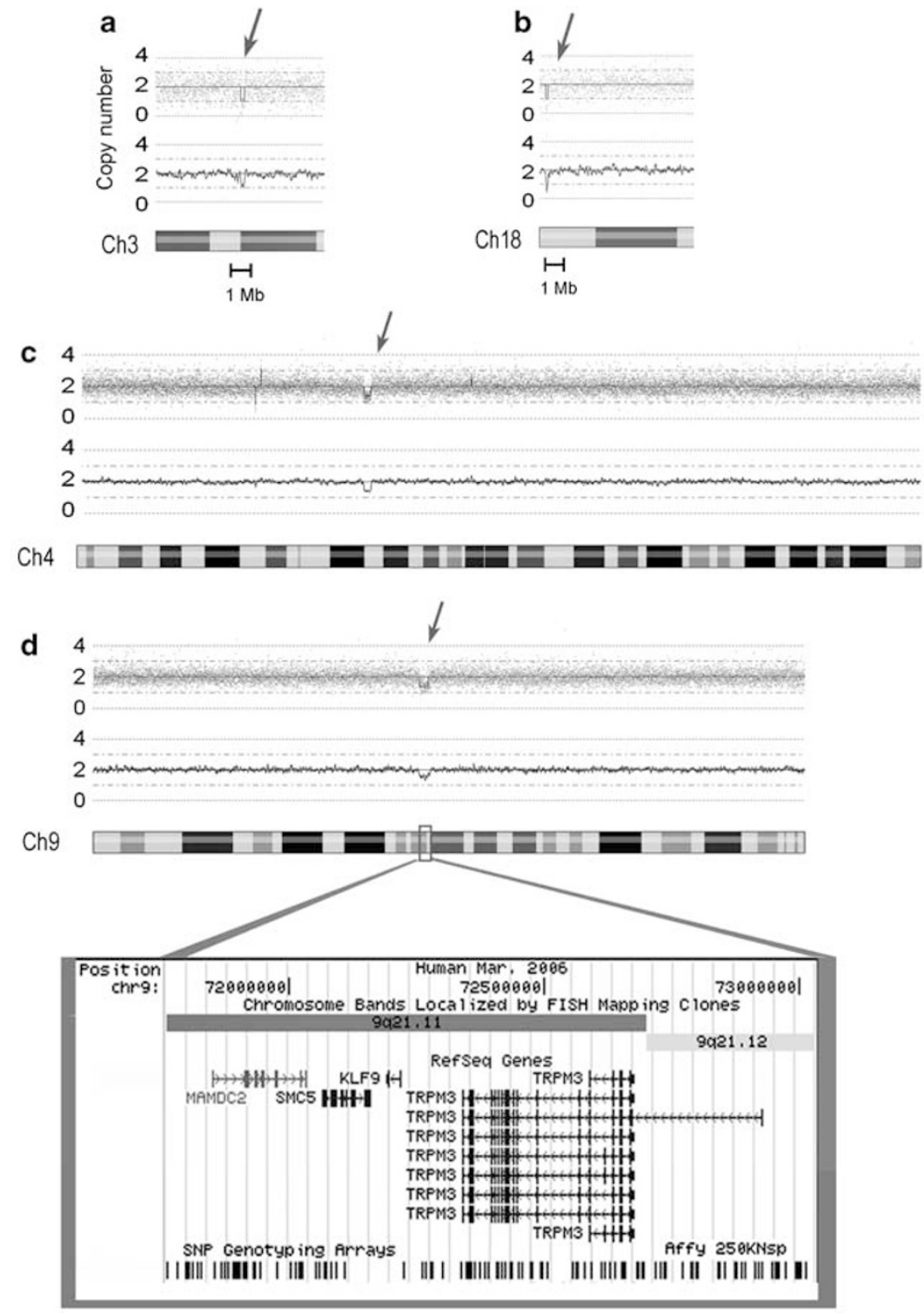

Figure 1 Chromosome view of Copy Number Analyser for GeneChip (CNAG) analysis. Each dots represent fluorescent intensity on each single-nucleotide polymorphisms (SNP) probe of GeneChip 250K Nspl array (Affymetrix). Solid lines indicate copy number analyzed with CNAG. Arrows show detected deletions. (a) Chromosome (Ch) 3 of patient K9, $205 \mathrm{~kb}$ deletion in 3p26.2 involving an exon of SUMFlgene. (b) Chromosome 18 of patient K34, $\sim 35 \mathrm{~kb}$ deletion in 18p11.32, containing the CETN1 gene. (c) Chromosome 4 of patient $\mathrm{K} 23, \sim 1.26 \mathrm{Mb}$ deletion in $4 \mathrm{q} 13.2$, not involving any known gene. (d) Chromosome 9 of patient K16, $\sim 1.27 \mathrm{Mb}$ deletion in 9q21.11-q21.12, harboring four genes: TRPM3, KLF9, SMC5 and MAMDC2. The University of California Santa Cruz genome browser denotes the cytobands, genes and probe setting of Affymetrix 250K Nspl array within the region. No copy number variation was registered here in the Database of Genomic Variants updated 26 June 2008. FISH, fluorescent in situ hybridization. 


\section{RESULTS}

Molecular karyotyping and validation of deletion

The entries of molecular karyotyping were 18 patients with KS (K1, K3, K5, K6, K7, K8, K9, K11, K12, K13, K16, K18, K20, K21, K22, K23, $\mathrm{K} 34$ and $\mathrm{K} 38$ ). We eliminated the data of patient K3 from copy number analysis, because it showed low quality data; that is, a singlenucleotide polymorphism (SNP) call rate of $82.51 \%$ and a quality control performance detection rate of $74.09 \%$, probably because of DNA degradation during long-term storage. The other patients showed high call rates, enough for copy number analysis (SNP call rate of $90.07-97.72 \%$ and detection rate of $91.52-99.77 \%)$. We identified nine deleted regions, the lengths of which were between $\sim 35 \mathrm{~kb}$ and $\sim 1.27 \mathrm{Mb}$, and nine duplicated regions, of lengths between $\sim 72$ and $\sim 495 \mathrm{~kb}$, in the 17 patients analyzed (Table 1). As for the nine duplications detected, five of them were concordant to several observed gains in DGV, and four of them in each patient did not contain any known genes.

It is interesting that the deleted region of $9 \mathrm{q} 21.11-\mathrm{q} 21.12$ $(\sim 1.27 \mathrm{Mb}$ in patient K16), which had not been registered in DGV, harbored four known genes: transient receptor potential cation channel, subfamily M, member 3 (TRPM3), Kruppel-like factor 9 (KLF9), structural maintenance of chromosomes protein 5 (SMC5) and MAM domain containing 2 (MAMDC2) (Figure 1d). The deletion of 3p26.2 ( $\sim 205 \mathrm{~kb}$ in patient K9, Figure 1a) had involved a noncoding exon of the SUMF1 gene. The deletion of $18 \mathrm{p} 11.32(\sim 35 \mathrm{~kb}$ in patient K34, Figure 1b) containing the CETN1 gene had one registration in DGV as Variation_5044, which described only one observed loss and 14 observed gains in 95 individuals. The deletion of $4 \mathrm{q} 13.2$ $(\sim 1.26 \mathrm{Mb}$ in patient K23, Figure $1 \mathrm{c})$ and $20 \mathrm{p} 12.1(\sim 152 \mathrm{~kb}$ in patient K6) did not carry any coding exon of any gene. The regions of $14 \mathrm{q} 11.2(\sim 116 \mathrm{~kb}$ in patient $\mathrm{K} 5)$ and $15 \mathrm{q} 11.2(\sim 972 \mathrm{~kb}$ in patient $\mathrm{K} 1$ and $\mathrm{K} 23$ ) were non-pathological deletions with as many registrations as observed losses in DGV.

To validate the deletion of the detected region, we confirmed the loss of heterozygosities of the SNP probes present there using GTYPE (data not shown) and carried out qPCR. The regions of SUMF1 on 3p26.2 (for patient K9) and of MAMDC2 on 9q21.11-q21.12 (for patient K16) had one copy in each patient compared with those in unaffected individuals (Figure 2). The deletion of CETN1 on 18p11.32 (for patient K34) was inherited from his unaffected mother. As samples from the parents of patient K16 were unavailable, it was not possible to examine whether the deletion of $9 \mathrm{q} 21$ was de novo. But the deletion was not found in 95 normal Japanese individuals using qPCR (data not shown).

As a consequence of this copy number analysis, we considered the next four genes as candidate genes for KS: TRPM3, KLF9, SMC5 and $M A M D C 2$.

\section{Mutation screening and in silico analysis}

Table 2 shows the results from mutation screening of the four candidate genes in 41 patients with KS. Ten base substitutions were found in the 41 patients, consisting of six registered SNPs, one unregistered SNP and three silent mutations. In addition, SUMF1 (NM_182760) and CETN1 (NM_004066) were also screened, but no mutations were detected (data not shown).

We checked the three silent mutations for splice site alteration using the GeneSplicer program, but no activation of the cryptic splice site was predicted. Although PANTHER classification of the four candidate genes did not show significant correlation for biological processes or pathway because of its small scale in number, some genes associated with developmental biology;
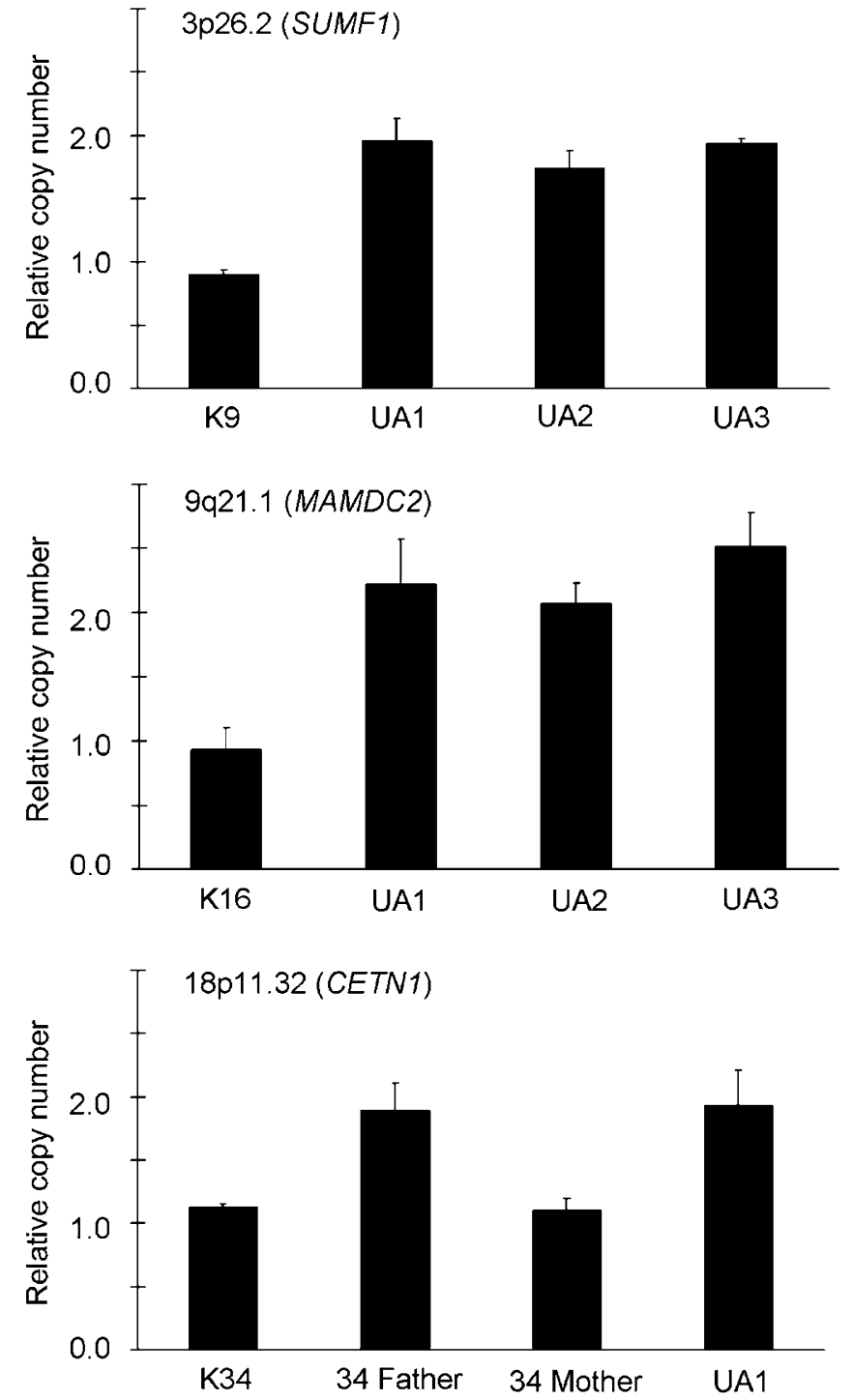

Figure 2 Validation of deletion with quantitative PCR (qPCR). $q P C R$ confirmed a loss of one copy in each patient: SUMF1 at 3p26.2 for patient K9; MAMDC2 at 9q21.1 for patient K16; CETN1 at $18 \mathrm{p} 11.32$ for patient $\mathrm{K} 34$. The deletion of patient K34 was inherited from his unaffected mother. UA, unaffected individual. Error bars, s.d.

that is, DNA repair (SMC5) and mRNA transcription regulation (KLF9).

\section{DISCUSSION}

We used high-resolution oligonucleotide array of GeneChip 250K NspI with a resolution of $30-100 \mathrm{~kb}$ and tried to find causative deletions or mutated genes for KS. Our molecular analysis did not strongly identify the causative gene for KS, but we identified a locus that possibly contributed to KS.

The deletion in patient K16, with a length of $\sim 1.27 \mathrm{Mb}$ at 9q21.11q21.12, harbored four known genes: TRPM3, KLF9, SMC5 and MAMDC2 (Figure 1d). Unfortunately, her parents' DNAs were unavailable, but the region is unlikely to be a copy number variant (CNV) because it has not been known as CNV in DGV; moreover, the deletion was not found in 95 normal Japanese individuals using qPCR.

As mutation screening in the 41 patients with KS showed no pathogenic base substitution in these genes, we cannot state that 
Table 2 Mutation screening of candidate genes in 41 patients with Kabuki syndrome

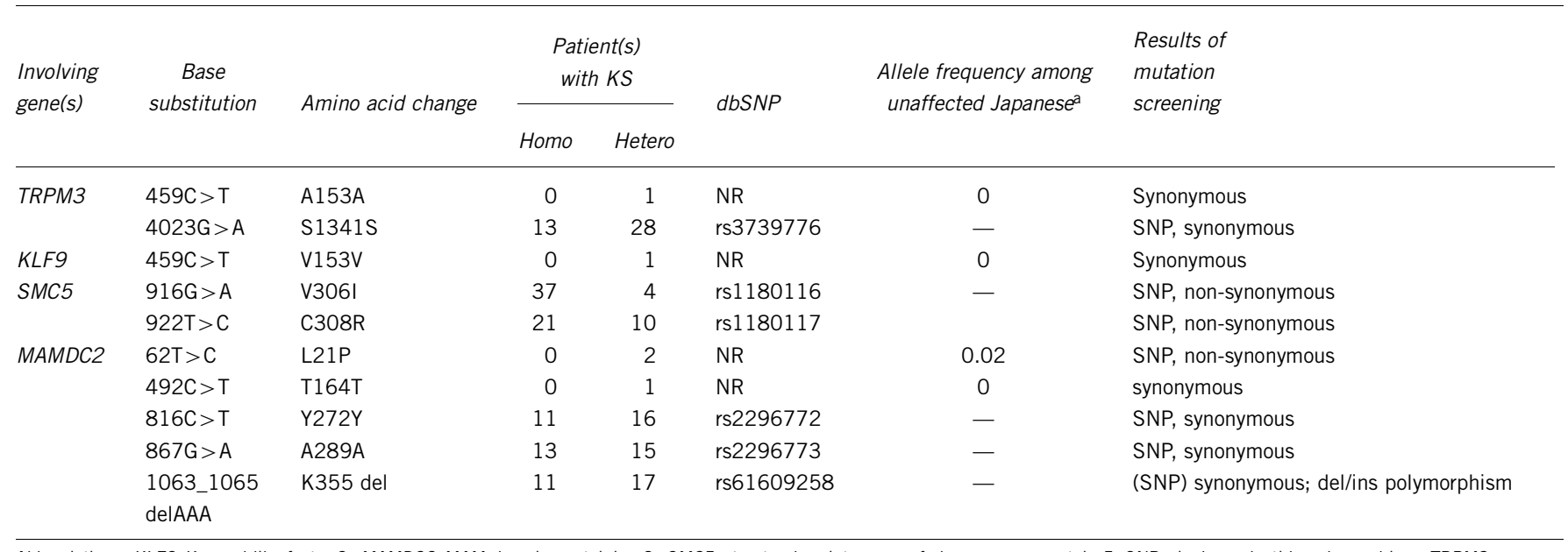

Abbreviations: KLF9, Kruppel-like factor 9; MAMDC2, MAM domain containing 2; SMC5, structural maintenance of chromosomes protein 5; SNP, single nucleotide polymorphism; TRPM3, transient receptor potential cation channel, subfamily M, member 3; dbSNP, registration number of database of SNP (http://www.ncbi.nlm.nih.gov/SNP/).

aAllele frequency was calculated from 188 chromosomes of 94 individuals.

these genes are major genetic factors for KS. However, it is presumable that the genes have some etiological roles for KS because of its genetic heterogeneity. Ontology of the PANTHER classification suggested that the three genes were associated with developmental biology, such as mRNA transcription regulation. Moreover, the $1.27 \mathrm{Mb}$ region of $9 \mathrm{q} 21$ was included in an earlier reported candidate locus of cleft lip/palate by meta-analysis of linkage analysis. ${ }^{24}$ Patient K16 actually had velopharyngeal insufficiency because of a submucous cleft palate. Therefore, it is reasonable to consider that the deleted genes cooperated with the development of a cleft palate, which is often accompanied by KS.

Although the $\sim 152 \mathrm{~kb}$ deletion within intron 5 of C20orf133 (MACROD2) in patient K6 did not involve any coding exon and her parents' DNAs were unavailable, the deletion was neither registered as CNV in DGV nor was it found in 95 normal Japanese individuals by qPCR (data not shown). Maas et al. ${ }^{14}$ reported de novo $\sim 250 \mathrm{~kb}$ deletion, including exon 5 of C20orf133 (MACROD2), in a patient with KS. Direct sequencing for the gene in 62 other patients with KS did not detect mutations, ${ }^{14,15}$ but the gene may be one of the causative genes for KS in consideration of its genetic heterogeneity.

We focused this study on KS on deletion/duplication detected using oligonucleotide array and mutation screening of the coding genes within the region. One limitation of this study is its resolution. As a matter of course, a higher resolution array can detect smaller genomic rearrangements, which were undetectable in the same patient, as we showed here compared with an earlier study of BAC array CGH. ${ }^{18}$ Although SNP probes are useful to examine loss of heterozygosity as a collateral evidence in deletions, unevenly distributed probes of the SNP array have a disadvantage for CNV detection. As various platforms on oligonucleotide array have developed, higher resolution platforms will have to be applied to search tiny genomic rearrangements in patients with KS. Another limitation is that we assumed that a single copy number change caused KS. It remains to be elucidated whether CNV association ${ }^{25}$ contributes towards manifestations of KS. If further investigation with refined array technologies cannot find the etiology of KS, the direction of study for KS will have to be changed to find de novo sequence alteration or methylation aberration, including in the non-coding genomic regions.

In summary, we applied molecular karyotyping with GeneChip $250 \mathrm{~K}$ array to detect copy number aberrations in 17 patients with KS and screened four candidate genes in 41 patients with KS. We could not identify causative DNA alteration for KS, but the locus, 9q21.11q21.12, including TRPM3, KLF9, SMC5 and MAMDC2, may contribute to the cleft palate of KS. Further investigations will be needed as various array platforms have the potential to specify genomic alterations for KS.

\section{ACKNOWLEDGEMENTS}

We are grateful to the patients and their parents for their participation in this research. We also thank Ms Yasuko Noguchi, Ms Miho Ooga and Ms Chisa Hayashida for their technical assistance. NN was supported in part by Grantsin-Aid for Scientific Research from the Ministry of Education, Sports, Culture, Science and Technology of Japan, and was supported by SORST from Japan Science and Technology Agency (JST) (Nos. 17019055 and 19390095, respectively). KY was supported in part by Grants-in-Aid for Scientific Research from the Ministry of Health, Labor and Welfare.

1 Niikawa, N., Matsuura, N., Fukushima, Y., Ohsawa, T. \& Kajii, T. Kabuki make-up syndrome: a syndrome of mental retardation, unusual faces, large and protruding ears, and postnatal growth deficiency. J. Pediatr. 99, 565-569 (1981).

2 Kuroki, Y., Suzuki, Y., Chyo, H., Hata, A. \& Matsui, I. A new malformation syndrome of long palpebral fissures, large ears, depressed nasal tip, and skeletal anomalies associated with postnatal dwarfism and mental retardation. J. Pediatr. 99, 570-573 (1981).

3 Niikawa, N., Kuroki, Y., Kajii, T., Matsuura, N., Ishikiriyama, S., Tonoki, H. et al. Kabuki make-up (Niikawa-Kuroki) syndrome: a study of 62 patients. Am. J. Med. Genet. 31, 565-589 (1988).

4 White, S. M., Thompson, E. M., Kidd, A., Savarirayan, R., Turner, A., Amor, D. et al. Growth, behavior, and clinical findings in 27 patients with Kabuki (Niikawa-Kuroki) syndrome. Am. J. Med. Genet. 127A, 118-127 (2004).

5 Matsumoto, N. \& Niikawa, N. Kabuki make-up syndrome: a review. Am. J. Med Genet. 117C, 57-65 (2003).

6 Wessels, M. W., Brooks, A. S., Hoogeboom, J., Niermeijer, M. F. \& Willems, P. J. Kabuki syndrome: a review study of three hundred patients. Clin. Dysmorphol. 11, 95-102 (2002).

7 Armstrong, L., Abd El Moneim, A., Aleck, K., Aughton, D. J., Baumann, C., Braddock, S. R. et al. Further delineation of Kabuki syndrome in 48 well-defined new individuals. Am. J. Med. Genet. 132A, 265-272 (2005).

8 Li, M., Zackai, E. H., Niikawa, N., Kaplan, P. \& Driscoll, D. A. Kabuki syndrome is not caused by a microdeletion in the DiGeorge/velocardiofacial chromosomal region within 22q 11.2. Am. J. Med. Genet. 65, 101-103 (1996).

9 Makita, Y., Yamada, K., Miyamoto, A., Okuno, A. \& Niikawa, N. Kabuki make-up syndrome is not caused by microdeletion close to the van der Woude syndrome critical region at 1q32-q41. Am. J. Med. Genet. 86, 285-288 (1999). 
10 Miyake, N., Harada, N., Shimokawa, O., Ohashi, H., Kurosawa, K., Matsumoto, T. et al. On the reported 8p22-p23.1 duplication in Kabuki make-up syndrome (KMS) and its absence in patients with typical KMS. Am. J. Med. Genet. 128A, 170-172 (2004).

11 Hoffman, J. D., Zhang, Y., Greshock, J., Ciprero, K. L., Emanuel, B. S., Zackai, E. H. et al. Array based CGH and FISH fail to confirm duplication of 8p22-p23.1 in association with Kabuki syndrome. J. Med. Genet. 42, 49-53 (2005).

12 Sanlaville, D., Genevieve, D., Bernardin, C., Amiel, J., Baumann, C., de Blois, M. C. et al. Failure to detect an 8p22-8p23.1 duplication in patients with Kabuki (NiikawaKuroki) syndrome. Eur. J. Hum. Genet. 13, 690-693 (2005)

13 Kimberley, K. W., Morris, C. A. \& Hobart, H. H. BAC-FISH refutes report of an 8p228 p23. 1 inversion or duplication in 8 patients with Kabuki syndrome. BMC Med. Genet. 7, 46 (2006).

14 Maas, N. M., Van de Putte, T., Melotte, C., Francis, A., Schrander-Stumpel, C. T., Sanlaville, D. et al. The C20orf133 gene is disrupted in a patient with Kabuki syndrome. J. Med. Genet. 44, 562-569 (2007).

15 Kuniba, H., Tsuda, M., Nakashima, M., Miura, S., Miyake, N., Kondoh, T. et al. Lack of C20orf133 and FLRT3 mutations in 43 patients with Kabuki syndrome in Japan. J. Med. Genet. 45, 479-480 (2008).

16 Cuscó, I., del Campo, M., Vilardell, M., González, E., Gener, B., Galán, E. et al. Array$\mathrm{CGH}$ in patients with Kabuki-like phenotype: identification of two patients with complex rearrangements including 2 q37 deletions and no other recurrent aberration. BMC Med. Genet. 9, 27 (2008).

17 Schoumans, J., Nordgren, A., Ruivenkamp, C., Brøndum-Nielsen, K., The, B. T., Annéren, G. et al. Genome-wide screening using array-CGH does not reveal microdeletions/microduplications in children with Kabuki syndrome. Eur. J. Hum. Genet. 13, 260-263 (2005).
18 Miyake, N., Shimokawa, O., Harada, N., Sosonkina, N., Okubo, A., Kawara, H. et al. No detectable genomic aberrations by BAC Array CGH in Kabuki make-up syndrome patients. Am. J. Med. Genet. 140A, 291-293 (2006).

19 Zhang, Z. F., Ruivenkamp, C., Staaf, J., Zhu, H., Barbaro, M., Petillo, D. et al. Detection of submicroscopic constitutional chromosome aberrations in clinical diagnostics: a validation of the practical performance of different array platforms. Eur. J. Hum. Genet. 16, 786-792 (2008).

20 Chen, Y., Takita, J., Choi, Y. L., Kato, M., Ohira, M., Sanada, M. et al. Oncogenic mutations of ALK kinase in neuroblastoma. Nature 455, 971-975 (2008).

21 Nannya, Y., Sanada, M., Nakazaki, K., Hosoya, N., Wang, L., Hangaishi, A. et al. A robust algorithm for copy number detection using high-density oligonucleotide single nucleotide polymorphism genotyping arrays. Cancer Res. 65, 6071-6079 (2005).

22 Monis, P. T., Giglio, S. \& Saint, C. P. Comparison of SYTO9 and SYBR Green I for realtime polymerase chain reaction and investigation of the effect of dye concentration on amplification and DNA melting curve analysis. Anal. Biochem. 340, 24-34 (2005).

23 Thomas, P. D., Campbell, M. J., Kejariwal, A., Mi, H., Karlak, B., Daverman, R. et al. PANTHER: a library of protein families and subfamilies indexed by function. Genome Res. 13, 2129-2141 (2003).

24 Marazita, M. L., Murray, J. C., Lidral, A. C., Arcos-Burgos, M., Cooper, M. E., Goldstein, T. et al. Meta-analysis of 13 genome scans reveals multiple cleft lip/palate genes with novel loci on 9q21 and 2q32-35. Am. J. Hum. Genet. 75, 161-173 (2004).

25 Franke, L., de Kovel, C. G., Aulchenko, Y. S., Trynka, G., Zhernakova, A., Hunt, K. A. et al. Detection, imputation, and association analysis of small deletions and null alleles on oligonucleotide arrays. Am. J. Hum. Genet. 82, 1316-1333 (2008).

Supplementary Information accompanies the paper on Journal of Human Genetics website (http://www.nature.com/jhg) 